\title{
Cystoid macular edema associated with limbal melanoma *
}

\author{
J. Reimer Wolter \\ Departments of Ophthalmology and Pathology, University of Michigan Medical Center, Ann Arbor, Michigan 48109, USA
}

\begin{abstract}
Early cystoid macular edema causing some loss of central vision in an eye with an 8-year history of limbal melanoma was studied histopathologically. A typical combination of slight chronic uveitis, vitreoretinal traction, parafoveal separation of posterior vitreous and inner-limiting membrane, swelling with distinct cystoid changes in Henle's fiber layer, and foldlike detachment of foveal cones is described and discussed.
\end{abstract}

\section{Introduction}

Cystoid macular edema (CME) usually occurs as a reaction to disease processes involving the inner eye. To the clinician CME is most important when it is seen following trauma or surgery $[5,9-13,17]$. However, CME was first recognized in retinitis pigmentosa [4]. It may also occur as an idiopathic condition [3], as a result of topical epinephrine [6] or systemic nicotinic acid medication [3], and in association with central retinal vein occlusion [7] or papilledema [14], for example. CME is a common complication of choroidal melanoma $[1,2,15]$. It has also been seen in a case of ciliary body melanoma [16].

The purpose of the present study is to present a histopathological study of typical early CME in an eye with a superficial limbal melanoma. This study gives morphological support to clinical observation of CME in other superficial involvements, such as CME after epinephrine drops [6] or after contact lens overwear (C.F. Bahn and R.C. Crossen, submitted for publication).

\section{Methods}

Case report. A 38-year-old white male was referred to me on 6 March 1979 with regrowth of a pigmented tumor on the limbus of his right eye. This had been observed and removed for the first time 5 years earlier. Regrowth had been very slow. On 6 March 1979 vision was a solid 20/20 in both eyes. The dark-brown tumor covered the limbal region between 10 and 1 o'clock and was associated with segmental increase of superficial perilimbal vascularity. The tumor and a layer of underlying superficial sclera and cor-

\footnotetext{
* Supported by the Research to Prevent Blindness, Inc., New York, N.Y., USA

Offprint requests to: J. Reimer Wolter, M.D.
}

nea, as well as surrounding conjunctiva, were removed surgically on 12 March 1979 . The tumor was histologically diagnosed as an active limbal nevus.

The patient was sent back to me on 29 February 1980. He had regrowth of a pigmented tumor in the limbal region of the same eye directly below the lower aspect of the previous limbal nevus at about 9 o'clock. Under my observation this lesion enlarged and developed increased superficial blood supply. Finally, the tumor was removed along with an underlying layer of sclera and surrounding conjunctiva on 29 November 1980. However, this time the tumor was more atypical and had to be classified as a malignant melanoma. The area of the tumor was watched clinically and regrowth of pigmented tumor became evident within a few months. After oncology consultation and careful general examination, which was negative, enucleation was advised. The enucleation of the right eye was done on 24 July 1981 along with removal of conjunctiva and Tenon's capsule in the region of the tumor.

Vision had been an easy 20/20 in both eyes, until the patient observed "a filmy spot in the center of the vision" in his right eye early in 1981. Central vision was found to be decreased to $20 / 25$ in the right eye at that time. Vision had further decreased to a difficult $20 / 30$ and to $20 / 40$ at one time in the right eye, directly before this eye was enucleated. Vision in the left eye remained normal. The central cornea of the right eye had remained clear and there were no clinical signs of uveitis. Unfortunately, a special effort to study the nature of the visual defect in the right eye was not made.

\section{Results}

Pathological description. The eye was fixed in 10\% buffered formalin immediately after its removal. It was of normal size and exhibited the dark-brown limbal tumor measuring $4 \times 3 \mathrm{~mm}$ at $3 \mathrm{o}^{\prime} \mathrm{Clock}$. When the eye was opened in a horizontal plane, the tumor was cut in half and it measured $0.4 \mathrm{~mm}$ at its greatest depth. The anterior segment was otherwise completely normal. The lens was in a normal position and it appeared grossly clear. The vitreous was in place and it was clear, but it was under tension in an anteroposterior direction towards central retina and optic nerve. A thin layer of nondistinct vitreous clouding was visible in the vitreous layers directly in front of the central retina and optic nerve. Swelling and irregular star-shaped folding 


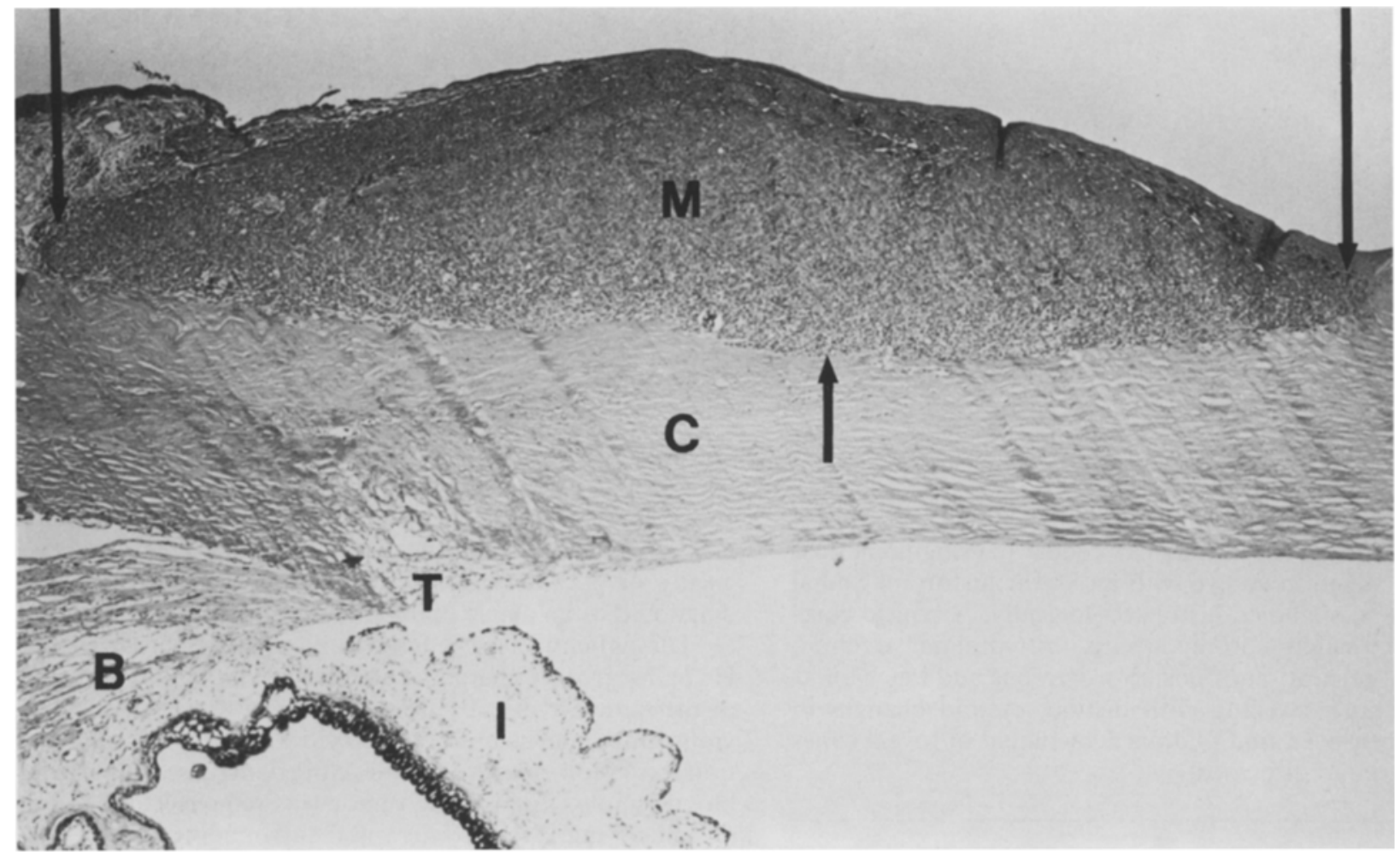

Fig. 1. Cross section of the limbal melanoma $(M)$ extending between the three arrows to about half of the thickness of the peripheral cornea $(C) . T$, uninvolved trabeculum and Schlemm's canal; $B$, ciliary body; $I$, iris. Paraffin section, H\&E stain, photomicrograph $\times 90$

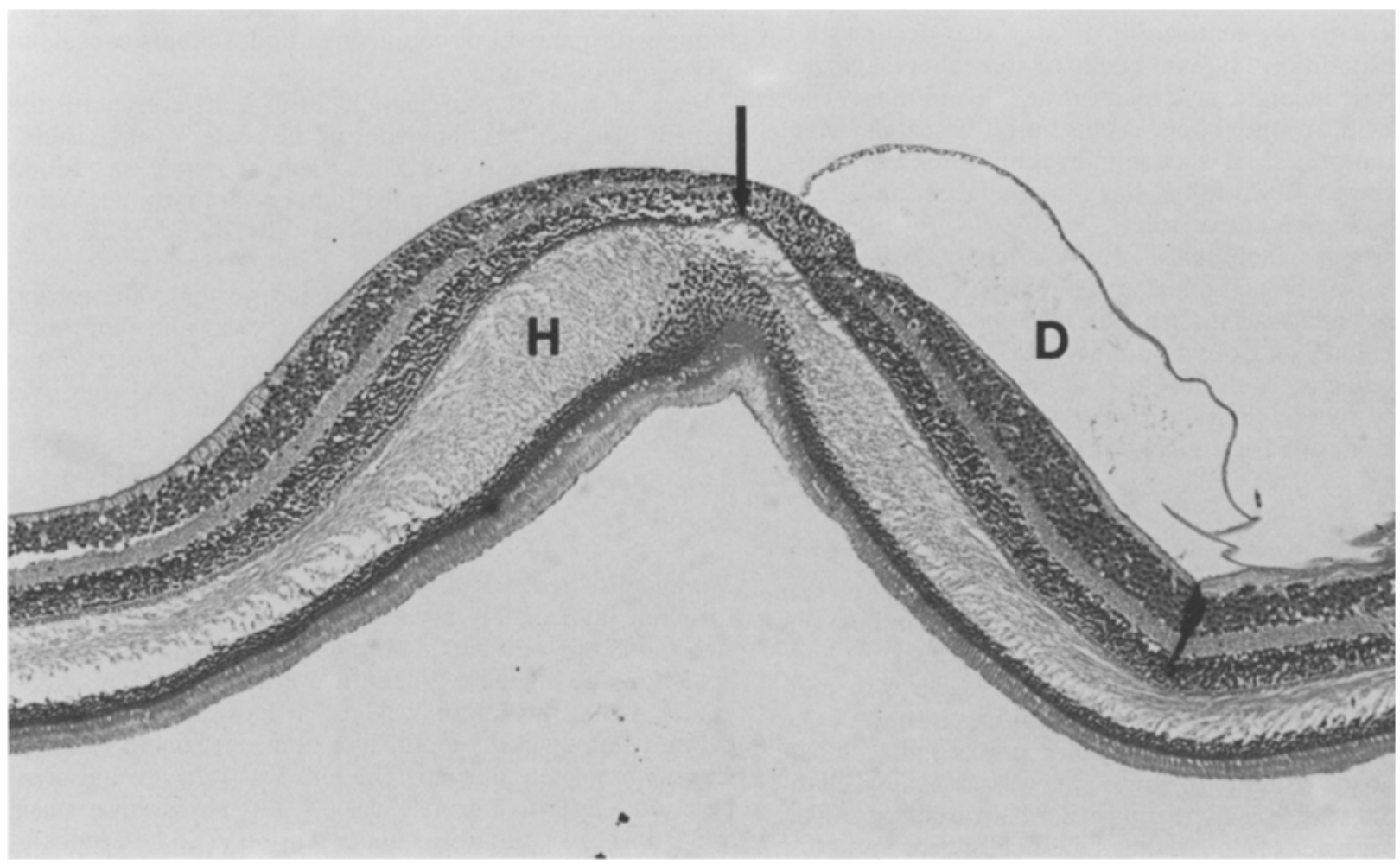

Fig. 2. The foveola in horizontal cross section slightly below the center with combined detachment of inner limiting membrane and posterior vitreous face $(D)$ containing a delicate serous exudate. Henle's fiber layer $(H)$ is swollen and its fibers are standing up. Cystoid spaces (arrow) are seen on top of the vertical fold running through the center of the foveola. - Paraffin section, $H$ and E stain, photomicrograph $\times 90$ 


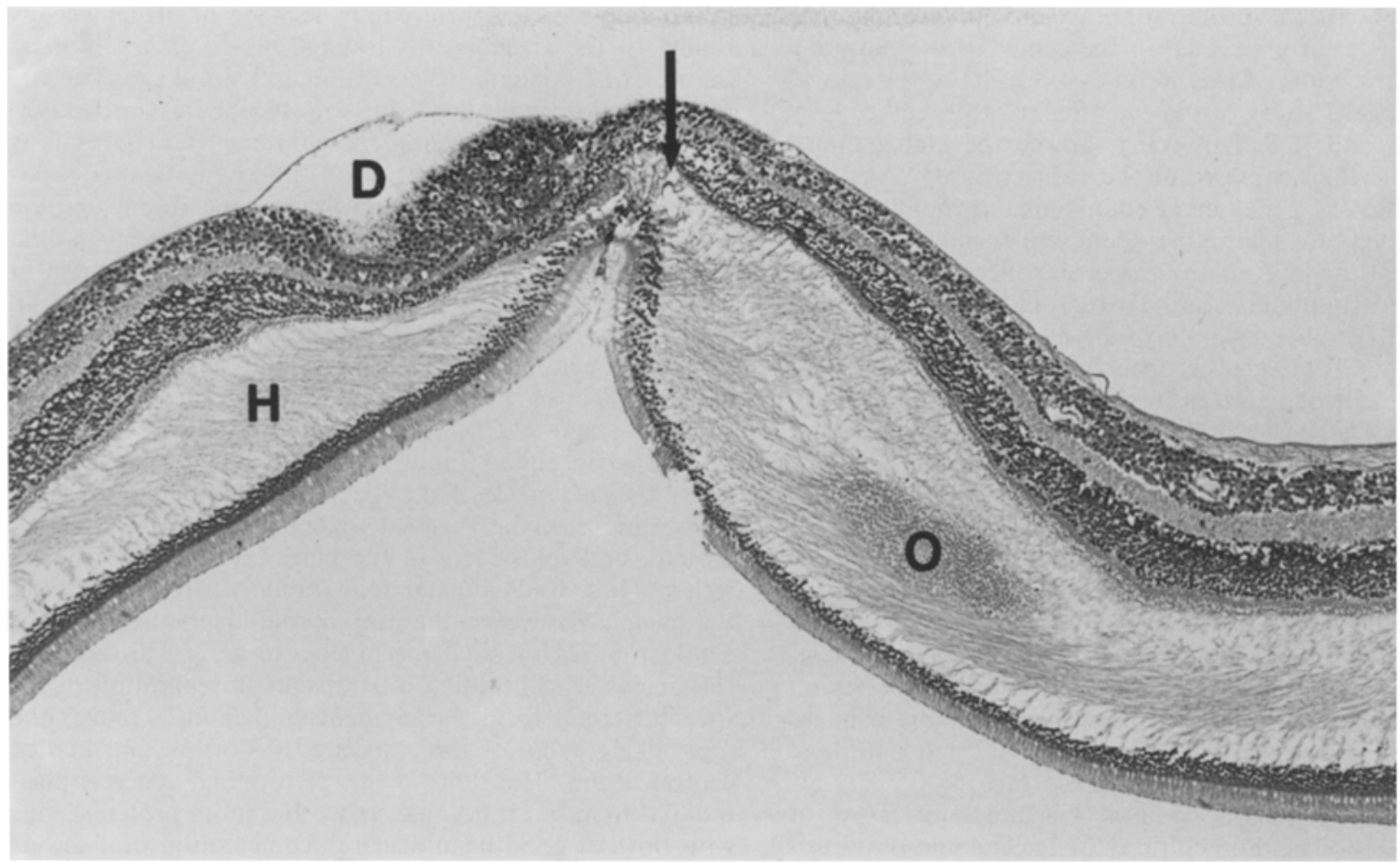

Fig. 3. The foveolar region slightly above the center with high vertical fold of outer retinal layers, swelling of Henle's fiber layer $(H)$, an oblique view of outer parts of the inner nuclear layer $(O)$, central cystoid degeneration (arrow), and separation of inner-limiting membrane and posterior vitreous face as a unit $(D)$ containing traces of a serous exudate. Paraffin section, H\&E stain, photomicrograph $\times 90$

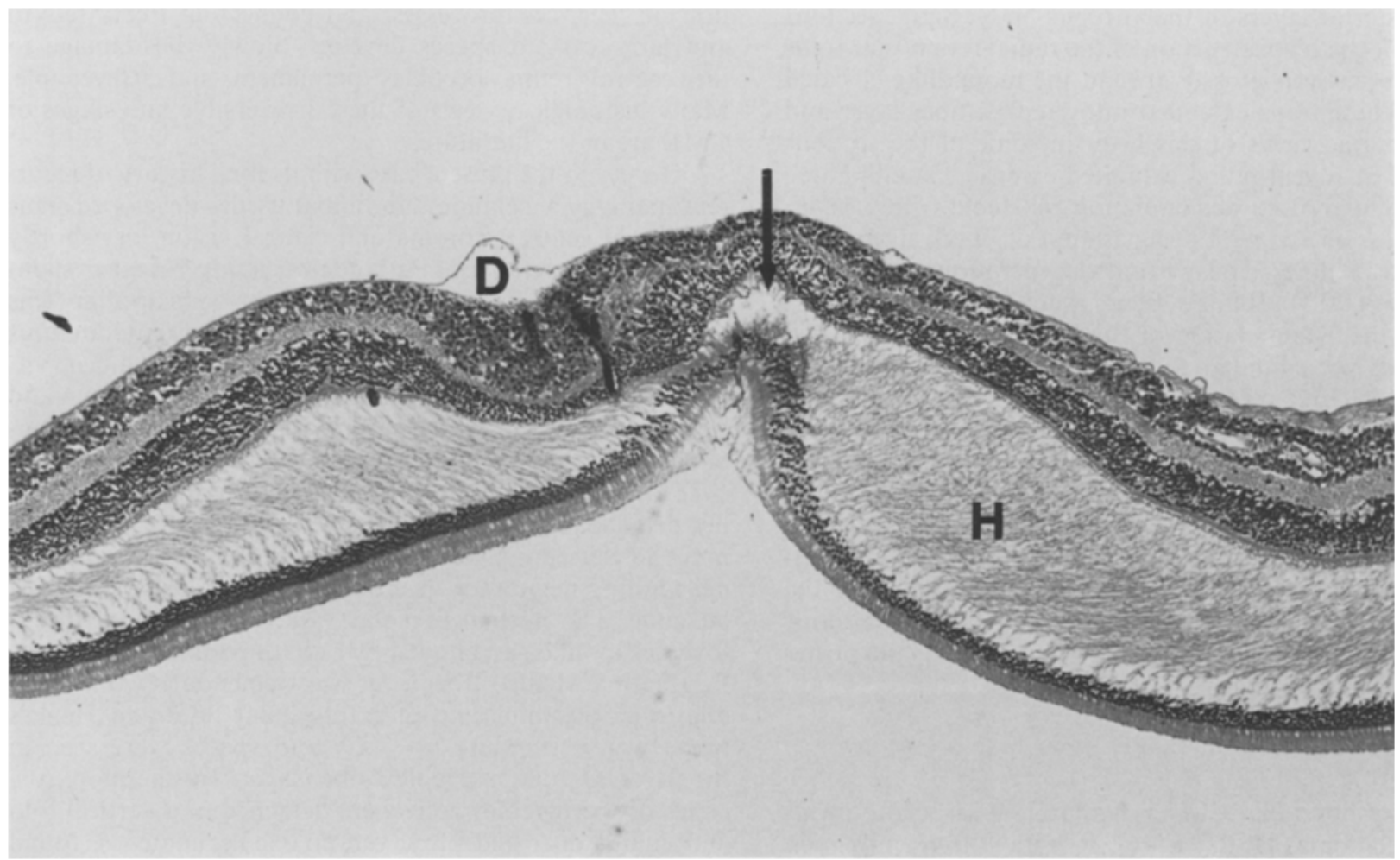

Fig. 4. Foveolar retina well above the center with slight degeneration of cones in the center of the high vertical fold, central cystoid degeneration (arrow), swelling of Henle's fiber layer $(H)$, and a small area of detachment of internal limiting membrane and posterior vitreous face $(D)$ from the inner retinal surface. Paraffin section, H\&E stain, photomicrograph $\times 90$ 
were grossly visible in the retina around the foveola. The foveola was clearly elevated in the center of the radiating folds of surrounding foveal retina.

Histological study of the eye after paraffin embedding, sectioning, and $\mathrm{H} \& \mathrm{E}$ staining showed the limbal tumor to resemble the neoplasm of the earlier biopsy. Again, it was classified as a malignant conjunctival melanoma. This had extended into the limbal sclera and cornea, but it had not reached the level of the trabecular meshwork (Fig. 1). Uninvolved conjunctiva and Tenon's capsule were seen all around the peripheral aspects of the sharply limited melanoma. The chamber angle was open, but there was much deposition of pigment granules in the trabecular endothelium. Iris and ciliary body exhibited slight and diffuse infiltration with occasional mononuclear inflammatory cells. A few small newly formed, thin-walled blood vessels were found in the superficial iris stroma. The pigment epithelium of the iris had diffuse cystoid changes with slight and irregular loss of pigment. There was one small posterior synechia of the iris segment next to the limbal tumor. The lens had very slight peripheral, posterior changes that were subcapsular and cataractous. The peripheral retina in the region of the vitreous base exhibited pronounced cystoid changes with accumulation of slightly basophilic substance in these cystoid spaces. The posterior hyaloid face was firmly attached to the retina all around. The posterior layers of the vitreous body exhibited preretinal accumulation of diffuse eosinophilic material resembling a trace of serous exudation. An irregular parafoveolar zone of the retina revealed an area of local separation of the posterior vitreous face, together with the inner limiting membrane from the remainder of the central retina (Figs. 2-4). The space under these separated layers contained denser condensations of the same kind of eosinophilic exudate as those observed in the posterior layers of the vitreous body. Serial sections allowed for the reconstruction of the radial retinal star folds that had been seen grossly around the moundlike elevated foveola. These folds extended into Henle's fiber layer and caused oblique views of this layer in some of the sections (Fig. 3). The foveal retina exhibited extensive swelling due to extracellular fluid accumulation in Henle's fiber layer. This was associated with degeneration of fibers, diffuse microcystoid changes, and cystoid degeneration in the very center (Figs. 2-4). Henle's fibers were "standing up" to allow for the accumulation of fluid in this layer. True cystoid spaces were limited to the foveolar zone (Fig. 4). A mild vertical fold of the outer retinal layers had formed and the cones of the center were not only elevated from the pigment epithelium, but also showed distortion and early degeneration. No trace of a fixed exudate was found in the retroretinal space. The pigment epithelium was normal. Surprisingly, one distinct focus of mononuclear infiltration without secondary scarring or degeneration of the overlying pigment epithelium was found in the posterior choroid slightly nasal to the disk. The optic nerve and posterior sclera were normal.

\section{Discussion}

Experience shows that CME is common in association with ocular melanomas $[1,2,15,16]$. It is most frequently seen in choroidal melanomas involving the foveal region of the choroid $[1,2,8,15]$. The reason is probably that ocular melanomas typically go along with some uveitis. The chron- ic inflammation, in turn, causes leakage of proteinaceous fluids. In the vitreous this leakage results in traction by the way of secondary degeneration and shrinkage. The vitreous traction typically is anteroposterior in its direction. When the posterior vitreous face still has firm connections to the inner-limiting membrane of the fovea, regional separation occurs between the inner-limiting membrane and the remainder of the retina. The separated layer overlying the central retina is sometimes ophthalmoscopically visible in eyes of young patients with CME. An exudate containing sufficient protein to allow for fixation by formaline is often found between the separated inner limiting membrane and the remainder of the retina [15].

Early stages of CME go along with extensive accumulation of extracellular fluid in Henle's fiber layer. Henle's fibers "stand up" to make space for this fluid. The foveal retina thickens, the foveolar valley turns into a foveolar mound, and folds form in the inner layers of the central retina. These folds are star folds radially arranged around the foveola. However, the deep retinal layers usually form a fold running through the foveola in a vertical direction [11]. This vertical folding often results in separation of the foveolar cones from the pigment epithelium - and is the most likely cause of the associated distortion and loss of central vision. The fluid in this retroretinal space is plasmoid at first, but it becomes more and more proteinaceous with time. A good histological documentation of a serous exudate under the detached central retina in CME was given in a recent publication [9] (Fig. 9), as well as in a recent paper by Kincaid et al. [5] (Fig. 6), for example. For some time the microarchitecture of the central retina allows for all these adjustments without serious loss of functionally important cells or fibers. Early CME, therefore, is clinically reversible. When the condition persists, however, degeneration of cells, cell processes, and connecting fibers sets in and large cystoid spaces develop. Slowly the damage to the central retina becomes permanent and irreversible. Many histological views of these irreversible late stages of CME are in the literature.

The eye in the present case with its long history of recurrent malignant melanoma in limbal tissues developed signs of a slight central scotoma and central vision loss shortly before it was removed. Pathological study revealed slight chronic iridocyclitis, minute posterior subcapsular lens changes, one small focus of chronic nongranulomatous posterior uveitis, and early cystoid macular edema. The vitreous was under grossly visible anteroposterior tension and clearly exerted pull on the central retina. The vitreous contained abnormal exudate without cells - mostly in a layer over the posterior pole. The posterior vitreous face was not detached from the retinal surface in itself, but its foveal pull had caused regional separation of vitreous face and inner-limiting membrane as a unit in an irregular parafoveolar zone. The normal foveolar valley was changed into a foveolar mound surrounded by radiating folds of the superficial retina. Henle's fiber layer was tremendously thickened due to an accumulation of extracellular fluid and Henle's fibers were "standing up." Cystoid spaces were seen in the foveolar zone of Henle's fiber layer. Furthermore, the cones of the foveolar zone were detached in a vertical fold and slightly distorted. However, no fixed exudate was found in the retroretinal space. The present eye is ideally suited to serve as an example for the histopathology of CME in an early stage. It can also be used to remind us that 
the eye reacts as a functional unit and, specifically, that a process in the region of the limbus can be the cause of chronic inflammation and a fluid-exchange disturbance in the most posterior part of the central retina. This suspicion has further support by the observation that contact lens overwear has been seen to be followed by reversible $\mathrm{CME}$ (C.F. Bahn and R.C. Crossen, submitted for publication). In view of the frequent use of the limbal zone of the eye for surgical incisions and for placement of more or less permanent sutures, the practical significance of these observations is obvious.

\section{References}

1. Von Domarus D, Hinzpeter EN (1978) Zystoides Makulaodem über einem malignen Melanom der Aderhaut. Klin Monatsbl Augenheilkd 172:751

2. Duke-Elder Sir St (1966) System of Ophthalmology. CV Mosby Co, St. Louis, $9: 851$

3. Gass JDM (1977) Stereoscopic atlas of macular diseases, ed 2, CV Mosby Co, St. Louis, p 260

4. Irvine AR (1976) Cystoid maculopathy. Surv Ophthalmol $21: 1$

5. Kincaid MC, Green WR, Iliff WJ (1982) Granulomatous reaction to Choyce style intraocular lens. Ophthalmic Surg 13:292

6. Kolker AE, Becker B (1968) Epinephrine maculopathy. Arch Opthalmol 79:552
7. Maumenee AE, Emery JM (1972) An anatomical classification of diseases of the macula. Am J Ophthalmol 74:594

8. Newell FW Ophthalmology, 4th ed. CV Mosby, St. Louis, p 313

9. Wolter JR (1981) The histopathology of cystoid macular edema. Graefe's Arch Clin Exp Ophthalmol 216:85

10. Wolter JR (1981) Vertical folds of central retina and choroid in sudden ocular decompression. Ophthalmic Surg 12:190

11. Wolter JR (1981) Foveal pathology following anterior segment injury, Ophthalmic Surg 12:661

12. Wolter JR (1981) Cystoid macular edema in vitreoretinal traction. Opthalmic Surg 12:900

13. Wolter JR (1982) Leaky cystoid macular edema attracting vitreous macrophages. Opthalmic Surg 13:968

14. Wolter JR (1982) Concentric microwaves of Henle's fiber layer: in association with cystoid macular edema. Graefe's Arch Clin Exp Ophthalmol 219:1

15. Wolter JR (1982) Three basic types of foveal involvement in choroidal melanomas, Graefe's Arch Clin Exp Ophthalmol 218:237

16. Wolter JR (1982) Foveal pathology in ciliary body melanoma. Opthalmic Surg 13:309

17. Wolter JR, Croasdale RE, Bahn CF (1980) Reactions to an anterior chamber lens - two years after implantation. Ophthalmic Surg 11:794

Received May 9, 1983 